\title{
A la recherche d'un paradigme de gestion de crise de la Covid-19 au Pérou
}

La construction d'une stratégie

Irène Valitutto

\section{(2) OpenEdition}

Journals

Édition électronique

URL : https://journals.openedition.org/echogeo/20283

DOI : $10.4000 /$ echogeo.20283

ISSN : 1963-1197

Éditeur

Pôle de recherche pour l'organisation et la diffusion de l'information géographique (CNRS UMR 8586)

Référence électronique

Irène Valitutto, "A la recherche d'un paradigme de gestion de crise de la Covid-19 au Pérou », EchoGéo [En ligne], Sur le Vif, mis en ligne le 04 janvier 2021, consulté le 24 août 2021. URL : http://

journals.openedition.org/echogeo/20283; DOI : https://doi.org/10.4000/echogeo.20283

Ce document a été généré automatiquement le 24 août 2021.

\section{(c) $)(9)$}

EchoGéo est mis à disposition selon les termes de la licence Creative Commons Attribution - Pas

d'Utilisation Commerciale - Pas de Modification 4.0 International 


\title{
A la recherche d'un paradigme de gestion de crise de la Covid-19 au Pérou
}

\author{
La construction d'une stratégie
}

Irène Valitutto

\section{Introduction}

Cet article traite de la stratégie de réponse à la crise sanitaire de la COVID-19 par les acteurs nationaux et locaux au Pérou. Il offre une lecture des mécanismes de réponse observés depuis la capitale, Lima, qui est à la fois l'endroit qui concentre le plus grand nombre de contaminations ${ }^{1}$ et le centre de gestion de crise pour l'ensemble du pays. Comme soulevé par de nombreux chercheurs au moment d'autres catastrophes, le rôle déterminant de la capitale face à l'urgence au Pérou relève d'un système très centralisé (Sierra, 2015 ; Delaître et al., 2018).

L'analyse que nous présentons ici a été réalisée grâce à des données recueillies « sur le vif ", durant le déploiement des mesures de sécurité mises en place après la déclaration de l'état d'urgence dans le pays. Celles-ci sont issues d'observations directes sur le terrain, entre mi-janvier et mi-avril 2020, de la lecture de la presse nationale et de documents produits par diverses institutions nationales et locales. L'ampleur et le caractère inédit des événements liés à cette crise sanitaire, ainsi que les difficultés à la décrire au moment même du déploiement des faits, et donc la partialité des informations (comme souvent dans le premier temps d'une crise), nous semblent des facteurs à mettre en avant pour souligner les limites de cette analyse. 


\section{Un point de vue privilégié sur le processus de construction d'une réponse à la crise}

L'information constitue un élément essentiel pour définir une stratégie de réponse dans la gestion de crise. En même temps, «l'insuffisance d'information et de connaissance est une constante dans toutes les perturbations" (Lagadec, 1991). L'indécision et les ajustements continus d'une stratégie de réponse dépendent du fait que l'information nécessaire à la décision est insuffisante ou pas assez fiable. Ce manque d'information ou son inexactitude peuvent générer, entre autres, désinformations et mécontentement, et surtout conditionner considérablement la réponse (Lagadec, 1991 ; Delaître et al., 2018).

Devant une maladie méconnue, l'information nécessaire pour construire une réponse concerne en grande partie la définition des caractéristiques de la maladie et celle des modalités de sa diffusion. Comme dans le cas de la COVID-19, il est nécessaire d'apprendre les caractéristiques du virus pour comprendre comment en contenir la transmission. De cette manière, la stratégie de réponse est définie au fur et à mesure des progrès scientifiques et, pour cette raison, nous pouvons parler de processus de construction d'une réponse. Celui-ci a une temporalité différente d'une stratégie face à un risque déjà connu, comme par exemple une réponse à la suite d'une catastrophe naturelle. Dans le cas de la COVID-19, il a fallu créer un ensemble de mesures spécifiques, ce qui en fait un processus qui demande un temps de réflexion, d'expérimentation et d'ajustement. Ainsi, l'acquisition fragmentée des informations insère la réponse à la crise engendrée par la COVID-19 dans un processus en devenir, qui nous fournit un point de vue privilégié pour saisir son élaboration ainsi que les enjeux qu'elle révèle.

\section{Pouvons-nous parler de modèle de réponse?}

Si toute crise a des spécificités liées à un contexte local et s'inscrit dans une spatialité définie, une pandémie comme celle à laquelle nous sommes confrontés nous oblige à réfléchir en termes de globalisation. La pandémie a suivi deux logiques de diffusion dans l'espace mondial: une traditionnelle par "proximité de voisinage" des pays limitrophes, mais aussi une diffusion réticulaire par "proximité fonctionnelle » tissée par la mondialisation entre des territoires éloignés géographiquement pourtant interdépendants ou associés (Carroué, 2020 ; Lussault, 2020 ; Mesclier, 2020). Ainsi nous avançons l'hypothèse qu'à une mondialisation de la crise semblerait correspondre une mondialisation de la réponse, puis des réponses. En effet, la première expérience de gestion de la crise en Chine a servi de modèle pour inspirer d'autres stratégies de réponse ailleurs, mais celles-ci se sont ensuite modifiées sous l'influence des contextes locaux.

Si nous mobilisons le terme de modèle ici, c'est pour mettre l'accent sur les acceptions d'imitation et de duplication d'un ensemble de règles et de normes. « Le modèle est ici entendu comme une doctrine d'action, un schéma de politique ou d'instrument censé servir de référence à l'élaboration des politiques nationales " (Chartier et Rivière, 2018). Il nous semble aujourd'hui possible de distinguer trois types de modèles de réponses majoritaires : celui du confinement, total ou partiel, à différents degrés, à différentes échelles géographiques et à différents moments, qui a été le plus répandu ; 
celui du contrôle individuel de la population, mis en place par exemple à travers une application mobile, comme en Corée; ou encore, comme en Suède, celui de la responsabilisation individuelle des citoyens, bien plus flexible que les deux premiers, qui se base depuis le début de la crise sanitaire sur le seul respect volontaire des gestes barrière. Ces trois modèles peuvent aussi se combiner, dans différentes temporalités de la crise. Nous pouvons saisir alors le scénario du confinement suivi, par exemple, par celui de la responsabilisation citoyenne (avec la mise en place des gestes barrière), en même temps que les individus pourraient être invités à mettre en place un modèle de contrôle à travers l'installation d'une application mobile sur leur smartphone. Chaque modèle correspond à des enjeux spécifiques, de la même manière qu'il est le résultat d'un environnement culturel, économique et politique situé.

De nombreux chercheurs analysent les logiques d'appropriation et de réinvention locale des modèles, en étudiant les rapports de force qui peuvent les modifier ou les altérer au cours de leur implantation dans différents environnements (Darbon, 2009 ; Peyroux, 2012). Ces études montrent aussi une évolution, depuis une perspective théorique dans les années 1960 vers une perspective technicienne, avec des modèles qui deviennent de plus en plus des boîtes à outils (ou paradigmes) dépolitisées à utiliser comme techniques de gouvernement (Darbon, 2009).

Existe-il des modèles propres à la gestion de crise? Le moment de l'urgence se transforme souvent en un moment de changements soudains, comme l'affirmation de nouveaux modèles, légitimés par des causes de «force majeure ». Revêt (2018) propose l'expression de "monde des catastrophes » pour décrire un ensemble d'acteurs, de normes, de bonnes pratiques et par extension nous pourrions dire de modèles, pour la gestion des risques, des crises et des catastrophes. Les rencontres d'acteurs d'horizons divers qui font partie de ce "monde» (bailleurs, agences d'assurance, consultants, professionnels d'ONG, diplomates, scientifiques, etc.) deviennent des laboratoires où ils développent des normes, des outils et un langage commun. Ainsi, ces acteurs diffusent sur la scène internationale des modèles (de bonnes pratiques), qui feront l'objet d'appropriation dans les politiques nationales, au sein desquelles ils seront transformés et ensuite institutionnalisés. Nous chercherons alors à comprendre de quelle manière et selon quels facteurs du contexte péruvien, le modèle de réponse à la crise de la COVID-19 se transforme en lien avec son ancrage territorial, où il prendra alors des significations spécifiques par rapport à d'autres géographies.

\section{L'arrivée de la COVID-19 au Pérou}

"Quand l'épidémie de Covid-19 s'est déclenchée, le Pérou était déjà en état d'urgence sanitaire face à la dengue ${ }^{2}$ (plus de 4000 cas à la fin janvier). Dans ce pays les unités de soins intensifs du secteur public étaient déjà occupées à $90 \%$ de leur capacité avant l'arrivée du SRAS-CoV-2 révélant parallèlement une forte inégalité dans l'accès aux soins » (Sierra, 2020). Face à cette situation, en tenant compte des expériences des pays asiatiques et européens, ainsi que de la fragilité de son propre système de santé publique, le Pérou a décidé de mettre en place une politique de prévention précoce et drastique (Sierra, 2020). Ainsi, si la France enregistre le premier cas le 24 janvier et déclare la décision du confinement le 16 mars, lorsque le pays franchissait le seuil de 1861 cas et 61 décès, le Pérou a décidé de se confiner quand il n'y avait que 71 cas diagnostiqués (et pas de décès) sur le territoire national et quelques jours seulement 
après avoir repéré le premier cas de COVID-19, le 6 mars. Le 15 mars, le Président a proclamé à la fois la fermeture des frontières et une quarantaine obligatoire de 15 jours, qui a été prolongée à plusieurs reprises jusqu'à fin juin (soit un total de trois mois et demi). Malgré l'application de cette politique de prévention, le Pérou est devenu rapidement un des pays les plus touchés par l'épidémie. Selon les comptes du ministère de la Santé, à la date du 15 septembre 2020, le pays enregistre plus de 750000 cas de contamination et environ 31000 décès, avec une concentration de cas dans la capitale (illustration 1).

Illustration 1 - Données diffusées sur l'avancement de l'épidémie

\begin{tabular}{|c|c|c|c|c|c|}
\hline Pais & $\mathrm{PCR}(t)$ & $\begin{array}{c}\text { PRUEBA } \\
\text { RAPIDA(t) }\end{array}$ & $\begin{array}{l}\text { TOTAL } \\
\text { CASOS }(t)\end{array}$ & $\begin{array}{l}\text { FAllEO } \\
\text { DOS }\end{array}$ & $\begin{array}{l}\text { LETAUDAD } \\
\qquad(\%)\end{array}$ \\
\hline PERÚ & 176641 & 579771 & 756412 & 31283 & $4.14 \%$ \\
\hline AMAZONAS & 1024 & 11963 & 12987 & 204 & $1,57 \%$ \\
\hline ANCASH & 3820 & 17415 & 21235 & 1248 & $5,88 \%$ \\
\hline APURIMAC & 546 & 3654 & 4200 & 79 & $1,88 \%$ \\
\hline AREQUIPA & 4726 & 34320 & 39046 & 1297 & $3,32 \%$ \\
\hline AYACUCHO & 2310 & 8100 & 10410 & 286 & $2,75 \%$ \\
\hline CAJAMARCA & 2812 & 15621 & 18433 & 441 & $2,39 \%$ \\
\hline CALLAO & 7559 & 23171 & 30730 & 1687 & $5,49 \%$ \\
\hline cusco & 5782 & 13353 & 19135 & 387 & $2,02 \%$ \\
\hline HUANCAVELICA & 788 & 5209 & 5997 & 102 & $1,70 \%$ \\
\hline HUANUCO & 1350 & 13569 & 14919 & 366 & $2,45 \%$ \\
\hline ICA & 3312 & 21966 & 25278 & 1546 & $6,12 \%$ \\
\hline JUNIN & 2246 & 15745 & 17991 & 723 & $4,02 \%$ \\
\hline LA LIBERTAD & 3520 & 23195 & 26715 & 2158 & $8,08 \%$ \\
\hline LAMBAYEQUE & 2520 & 22191 & 24711 & 1650 & $6,68 \%$ \\
\hline $\begin{array}{l}\text { LIMA } \\
\text { METROPOLITANA }\end{array}$ & 116121 & 206019 & 322140 & 12703 & $3,94 \%$ \\
\hline LIMA REGIÓN & 5733 & 21294 & 27027 & 1306 & $4,83 \%$ \\
\hline LORETO & 3188 & 14318 & 17506 & 940 & $5,37 \%$ \\
\hline MADRE DE DIOS & 900 & 6466 & 7366 & 136 & $1,85 \%$ \\
\hline MOQUEGUA & 432 & 11271 & 11703 & 253 & $2,16 \%$ \\
\hline PASCO & 293 & 4364 & 4657 & 100 & $2,15 \%$ \\
\hline PIURA & 1053 & 32246 & 33299 & 1911 & $5,74 \%$ \\
\hline PUNO & 759 & 10829 & 11588 & 278 & $2,40 \%$ \\
\hline SAN MARTIN & 2624 & 13673 & 16297 & 686 & $4,21 \%$ \\
\hline TACNA & 1513 & 9499 & 11012 & 194 & $1,76 \%$ \\
\hline TUMBES & 697 & 6038 & 6735 & 293 & $4,35 \%$ \\
\hline UCAYALI & 1013 & 14282 & 15295 & 309 & $2,02 \%$ \\
\hline
\end{tabular}

Traduction de la terminologie utilisée dans la case en rouge : pays, test virologique (PCR), test antigénique (test rapide), total de cas de contamination, nombre de décès, taux de mortalité

Source : Site officiel du ministère de la Santé péruvien, 15 septembre 2020 - URL: https:// covid19.minsa.gob.pe/sala_situacional.asp

Dans la suite de ce texte, nous proposons de donner une lecture des événements qui ont suivi la déclaration d'état d'urgence au Pérou. En la considérant comme un processus, nous observons la manière dont la stratégie de réponse à la crise a été construite. Ainsi, cette analyse porte, d'un côté, sur l'étude du discours officiel, ici envisagé comme un des outils de définition et de construction de la (stratégie de) réponse, et de l'autre côté, sur l'observation des événements qui se sont déroulés en parallèle. En questionnant le processus de définition d'une réponse et en soulignant des dissociations qui ont parfois eu lieu entre le discours et les faits, nous cherchons à faire ressortir les risques et les opportunités que la crise semble mettre en évidence. Une situation de crise est un moment d'observation privilégié de certaines relations de pouvoir, car des décisions doivent être prises rapidement et dans l'incertitude. Ainsi, la particularité de la situation révèle des rapports de pouvoir qui sont peu visibles dans des conditions ordinaires. 


\section{À travers le prisme du discours officiel}

Le Président péruvien s'adresse de façon quotidienne (à quelques rares exceptions près) aux citoyens durant plus de deux mois dans la période suivant la déclaration de l'état d'urgence national. Ainsi, chaque jour, il les informe du nombre de personnes touchées par le virus et il communique les nouvelles mesures de sécurité à adopter. Ce discours est ici identifié comme un des outils de construction du modèle de gestion de crise COVID-19. Nous allons l'étudier pour chercher à en comprendre les enjeux.

Comme le dit Gilbert (1992), la gestion de crise et l'état d'urgence sont souvent pour les acteurs au pouvoir des occasions d'affirmer leur légitimité à l'échelle nationale. Ainsi, les événements que nous analysons ici semblent faire écho à ceux décrits par Sierra (2007) à propos du Président par intérim équatorien Alarcón face à la catastrophe de El Niño en 1998. Vizcarra, comme Alarcón, est un Président qui prend ses fonctions en tant que chef d'État dans des conditions particulières. En mars 2018, Kuczynski, accusé de corruption, annonce sa démission, Vizcarra lui succède. Dans ce cadre, la crise actuelle représente pour le Président péruvien une occasion d'affirmer sa légitimité et son autorité face à la population, en incarnant le "rôle de protecteur de la nation " (Sierra, 2007). Comme dans le cas de l'Équateur, la proclamation de l'état d'urgence survient avant que n'ait lieu la crise. Dans les deux cas, c'est l'attente de la catastrophe qui engendre un processus de légitimation de la part du chef de l'État.

«La proclamation de l'état d'urgence est pour le Président, en cas de crise grave, l'outil constitutionnel par excellence qui lui permet d'accroître considérablement son pouvoir " (Sierra, 2007). Ainsi, l'institutionnalisation de la gestion de crise devient un véritable enjeu de pouvoir. Le Président péruvien entouré par ses collaborateurs de différents échelons gouvernementaux (l'intégralité du conseil des ministres, des représentants de l'assemblée générale des gouvernements régionaux et le Président de l'association des municipalités du Pérou ${ }^{3}$ ) au moment de la proclamation de l'état d'urgence (illustration 2), est une image symboliquement parlante, qui questionne ces enjeux de légitimité et de pouvoir. 
Illustration 2 - Le premier discours officiel « à la Nation » du Président péruvien du 15 mars 2020 : déclaration de l'état d'urgence

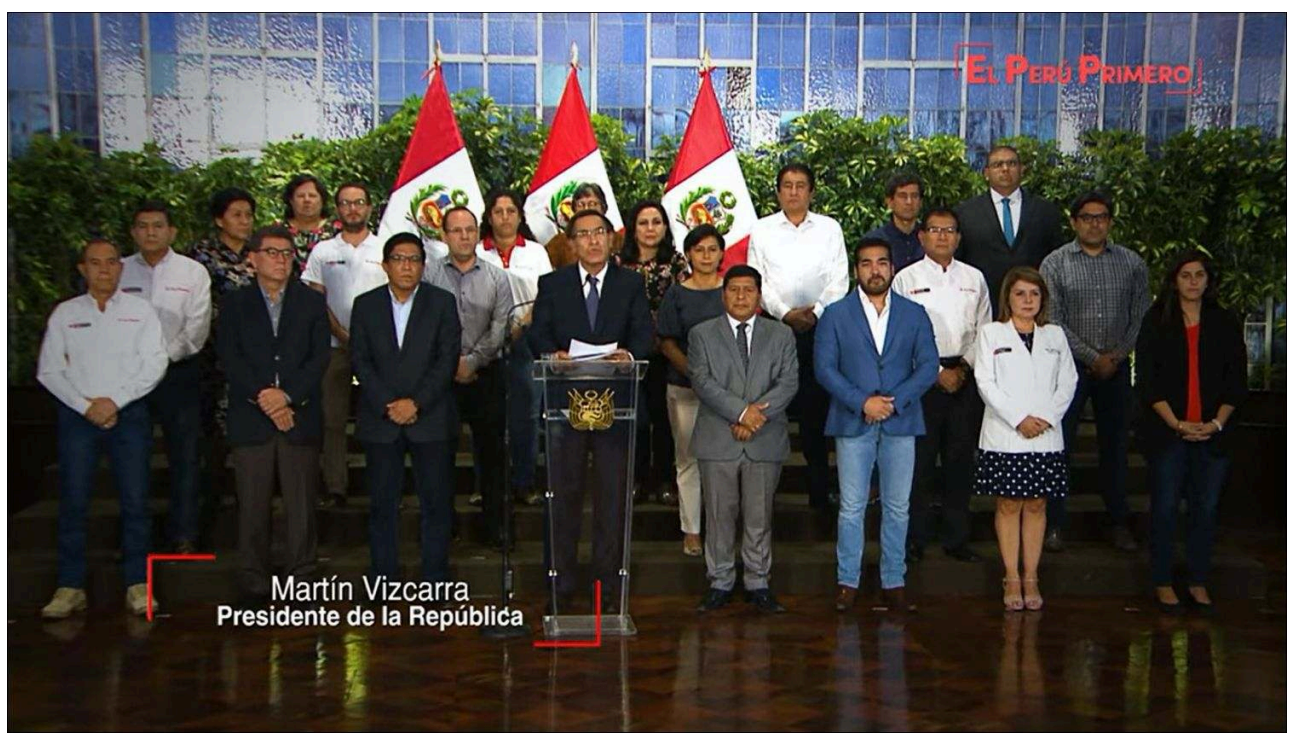

Source : URL: https://www.youtube.com/watch?

$\mathrm{v}=$ FoNmdCGZ4fO\&ab_channel=PresidenciaPer\%C3\%BA

\section{$1^{\text {er }}$ élément : le discours cherche une légitimité dans l'expérience des autres}

$\mathrm{Au}$ moment de la déclaration de l'état d'urgence dans le pays, le Président péruvien souligne dans son discours officiel la nécessité de s'inspirer d'expériences de succès d'autres pays. Il affirme : « Les pays qui ont assumé leur tâche de manière responsable face à cette maladie ont réussi. Les pays qui ne l'ont pas fait déplorent des quantités importantes de pertes humaines, des pertes économiques très importantes, mais surtout, un avenir incertain $»^{4}$. Il cherche ainsi à prendre en modèle des paradigmes de gestion utilisés par des pays qui sont parvenus à maîtriser la crise.

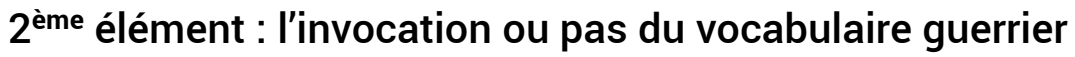

Contrairement à d'autres chefs d'État, comme par exemple le Président français pendant son discours du 15 mars 2020, le Président péruvien n'a pas mobilisé la métaphore d'une " guerre " pour décrire cette crise. Il se limite à dire que «la police nationale et les forces armées adopteront les mesures pour garantir les services publics» et que "pendant l'état d'urgence, les droits constitutionnels relatifs à la liberté et à la sécurité de la personne, à l'inviolabilité du domicile et à la liberté de réunion et de circulation sur le territoire national seront restreints $»^{5}$.

La formulation du discours semble éviter la rhétorique guerrière. Nous pourrions nous questionner sur les raisons qui expliquent le choix de ce vocabulaire. L'histoire de la République du Pérou a été très liée à celle de son armée : la plupart des Présidents qui ont gouverné, pendant 110 ans sur 179 d'indépendance du pays, ont été des militaires. Il y a eu 39 dirigeants l'État militaires contre 27 civils (Talavan, 2003). En outre, un conflit armé, qui a vu l'opposition des militaires avec le Sentier Lumineux, le mouvement révolutionnaire Tupac Amaru et des Rondas Campesinas (organisations 
autonomes d'autodéfense des paysans), a marqué l'histoire du pays entre les années 1980 et les années 1990. Ainsi, tout au long du XXe siècle, le pays voit la succession de huit coups d'État. Ce conflit est arrivé à terme pendant le mandat du Président Fujimori, figure emblématique dans le pays, qui se distingue par son régime autoritaire épaulé par les militaires et par d'importantes affaires de corruption ${ }^{6}$. La classe politique qui a pris le relais au XXI ${ }^{\mathrm{e}}$ siècle a fait aussi l'objet de nombreux scandales de corruption (en particulier « l'affaire Odebrecht $»^{7}$ à laquelle tous les derniers Présidents du pays sont liés : Garcia, Toledo, Humala, Kuczynski). Cependant, depuis la fuite et la démission de A. Fujimori, le Pérou est en train d'expérimenter une intense période de transition politique, qui a des répercussions à toutes les échelles du gouvernement (Talaván, 2003).

L'actuel Président entend prendre ses distances avec la classe politique liménnienne, qui est considérée comme la représentante de ce système centralisé et corrompu. Vizcarra semble relever le défi de recréer un lien de confiance avec la population, en basant son mandat sur la lutte contre la corruption. Dans ce cadre, son passé de gouverneur de la région périphérique de Moquegua (2011-2014), où il a su se distinguer par sa gestion des conflits avec les entreprises d'exploitation minière et la promotion d'une politique de développement régional, lui a fait gagner en légitimité au niveau national. Ainsi, son profil de technicien et d'homme politique originaire de l'intérieur du pays dessine une alternative à la classe politique de Lima, dans laquelle les Péruviens avaient perdu la confiance.

Dans ce contexte, depuis sa prise de fonction Présidentielle en 2018, Vizcarra s'est éloigné de toutes les factions politiques existantes. Ceci parce qu'il ne peut compter ni sur le soutien des personnalités traditionnelles du champ politique, ni sur les acteurs puissants du marché privé, qui sont méfiants vis-à-vis de lui et qui ne sont plus crédibles à cause de l'affaire Odebrecht. Il est en revanche soutenu par les forces armées du pays. Cette situation le porte ainsi à avoir recours directement et régulièrement au peuple dans sa stratégie politique (Mizrahi, 2019).

Pour le nouveau gouvernement, les enjeux de légitimation qui se cachent derrière la gestion de cette crise sanitaire sont donc nombreux. Le chef d'État semble vouloir tisser, dans ce moment singulier, un lien avec la population, en s'engageant à mener le pays au-delà de la crise en conservant une certaine transparence dans ses propos face aux citoyens. Ainsi, il affirme : « l'État est en train de respecter ses engagements (vis-àvis de cette crise) avec les institutions publiques, avec les familles et les personnes vulnérables. De nombreux entrepreneurs responsables ont pris des engagements aussi. Ce que nous demandons, c'est que nous nous engagions tous, cela dépend de nous. Nous pouvons lutter avec succès contre cette maladie difficile si nous nous engageons tous de manière responsable. Je fais pleinement confiance à la responsabilité de tous les Péruviens, je suis sûr que nous sortirons de cette situation compliquée de manière satisfaisante ${ }^{8} »$. Au fil de ses interventions quotidiennes, il semble chercher à construire un consensus et une confiance. Chaque jour, pendant environ une heure (ou plus), il informe le pays des nouvelles consignes à appliquer pour maîtriser la crise et ensuite il répond aux questions de représentants des médias tirés au sort. Souvent entouré de ses ministres pendant ces interventions, il les invite à répondre aux questions en fonction de leurs contenus spécifiques (santé, éducation, etc.). Cette démarche paraît relever des defis de transparence dans la gestion politique de la crise et lui permettre de consolider la confiance citoyenne. 


\section{Appropriation et transformation du modèle : vers une matrice militaire?}

Au prix d'une succession d'informations contradictoires, d'erreurs et d'ajustements, les modalités de gestion de la crise se façonnent jour après jour. Dès le début, les mesures adoptées semblent ne pas mener aux résultats attendus de baisse de cas de contamination, ce qui entraîne de nouvelles mesures pour renforcer le confinement : 15 jours après celui-ci, le 30 mars, le couvre-feu qui avait été fixé d'abord entre $20 \mathrm{~h}$ et $5 \mathrm{~h}$ passe au créneau $18 \mathrm{~h}-5 \mathrm{~h}$ ou $16 \mathrm{~h}-5 \mathrm{~h}$ selon les régions du pays ${ }^{9}$; pour limiter et mieux contrôler les flux de personnes dans les rues, les sorties pour des services essentiels comme les courses sont permises en alternance pour les femmes et les hommes, jours pairs pour les femmes et jours impairs pour les hommes, entre le 3 avril et le 12 avril 2020 ; début avril, les sorties sont interdites le dimanche pour l'intégralité de la population ${ }^{10}$ (c'est une mesure qui est toujours valable au moment de l'écriture de ce texte); durant la période de confinement, pour se déplacer en voiture ou en dehors du quartier de résidence, il est nécessaire d'avoir une attestation spéciale fournie par les institutions et les entreprises autorisées.

Illustration 3 - Communiqué officiel du gouvernement péruvien, concernant les nouvelles mesures prises face à l'état d'urgence de la COVID-19, avril 2020

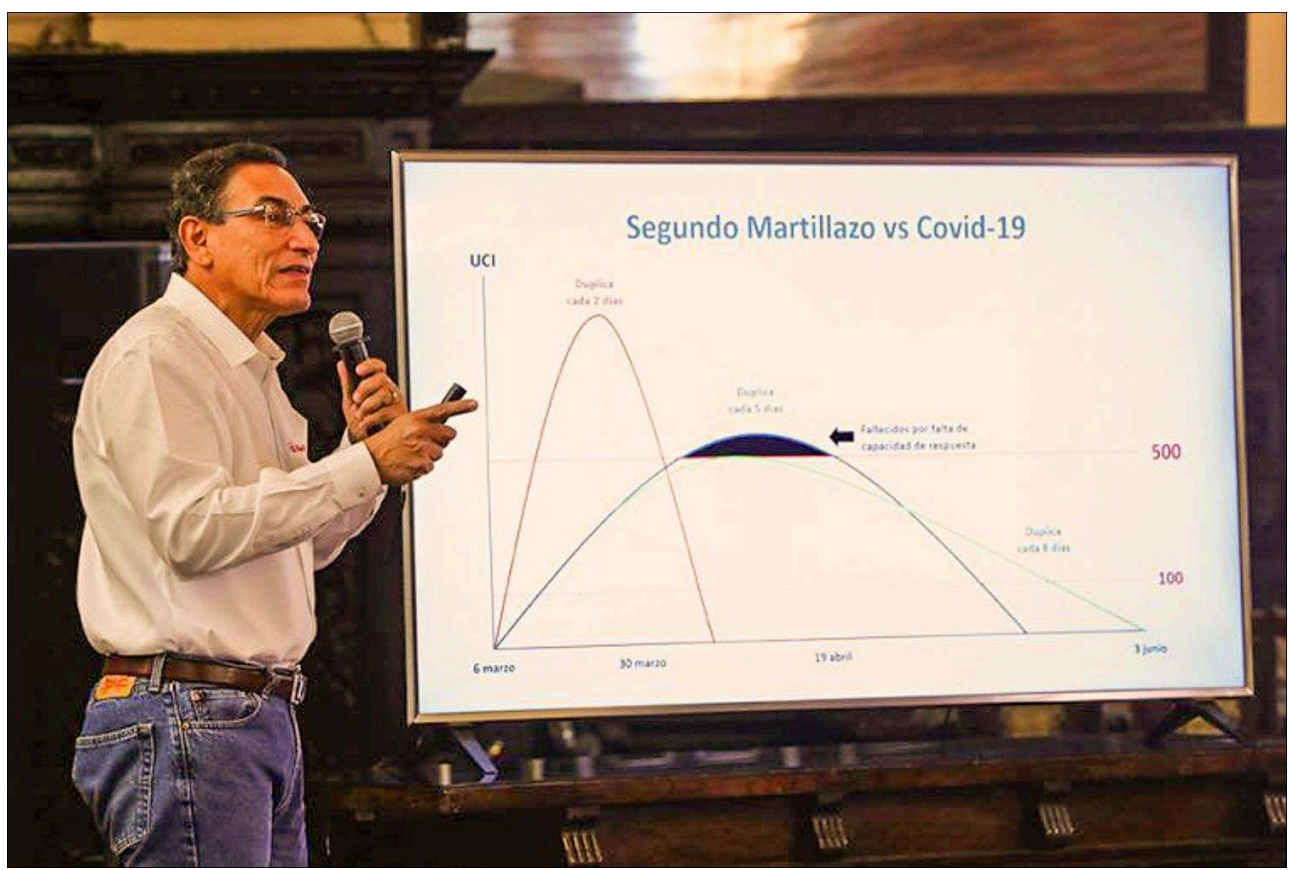

Source : https://www.elperuano.pe/noticia-hombres-y-mujeres-podran-circular-diasdiferenciados-93860.aspx

Le terme employé pour décrire ces mesure $s$ successives est le «martillazo» (illustration 3), faisant référence métaphoriquement à un «coup de marteau » à donner sur la courbe en hausse des cas de contamination, pour la faire baisser. Un coup de marteau après l'autre, le gouvernement remodèle et fait évoluer le modèle du confinement. Le langage choisi semble suggérer une idée de répression, qui pourrait faire écho aux règles de contrôle sévères imposées par les forces armées dans 
l'espace public. En déficit de personnel de surveillance, l'État a même sollicité l'aide d'anciens militaires ayant quitté leur service dans les deux dernières années pour reprendre exceptionnellement du service dans cette situation d'urgence ${ }^{11}$.

En décalage avec son premier discours à la Nation de mi-mars, les forces armées sont largement déployées sur le territoire depuis la déclaration de l'État d'urgence. Ainsi, dans la ville de Lima, certains quartiers sont considérablement militarisés ${ }^{12}$, avec une présence massive des soldats dans les rues (illustration 4). Que montre cette tension entre discours et action du point de vue de l'adaptation du modèle de réponse?

Illustration 4 - La police et l'armée contrôlent le respect de l'ordre dans l'espace public, face à l'entrée du supermarché Metro

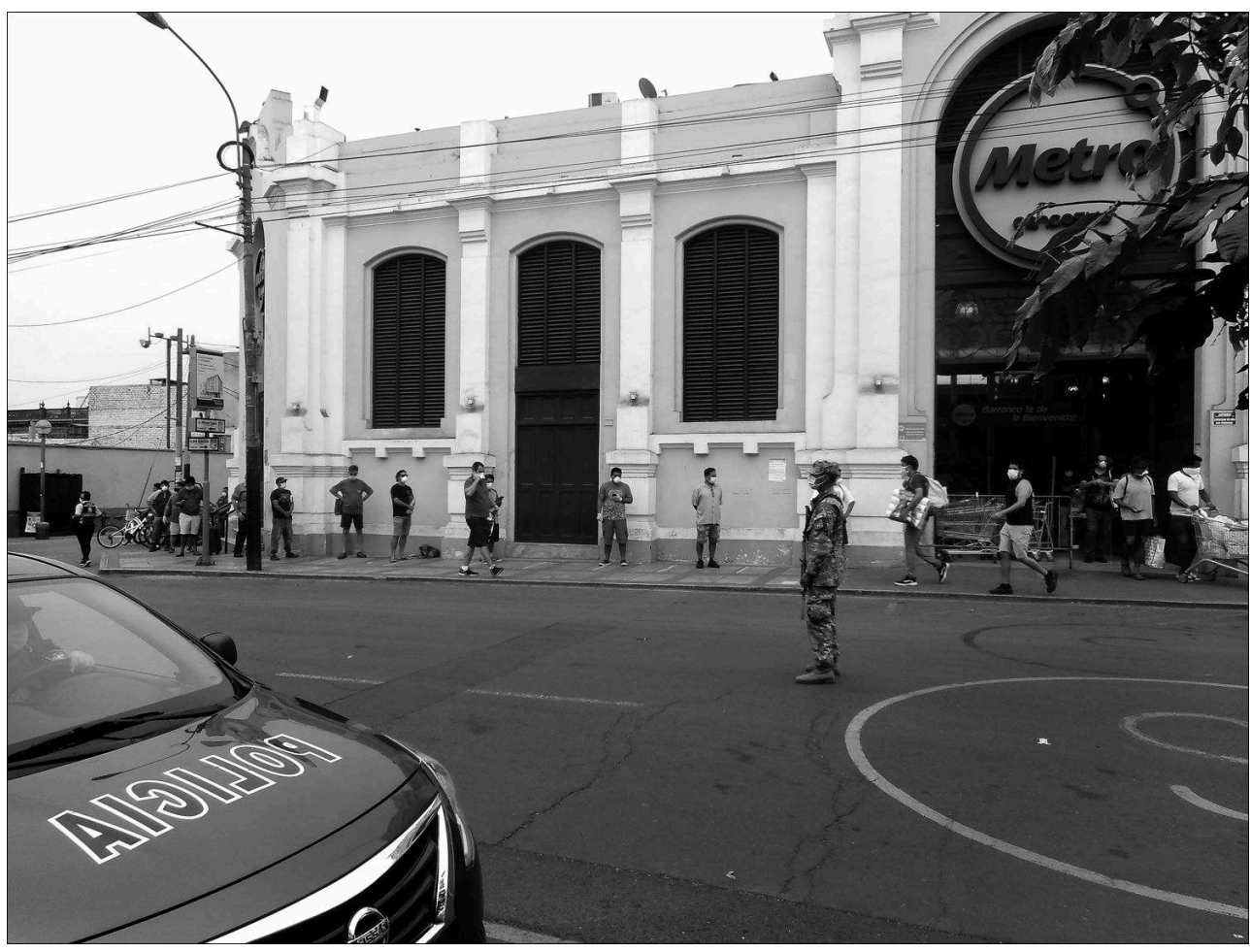

Auteure : I. Valitutto, Quartier de Barranco, Lima, avril 2020

Dans l'un de ses discours quotidiens, le Président affirme : «On demande l'attention de tous les citoyens. Réfléchissez bien deux fois avant de sortir de chez vous, parce que les contrevenants aux règles de confinement seront jugés par un procès pénal. Votre irresponsabilité ne restera pas impunie» ${ }^{13}$. Les mots mobilisés font référence aux concepts de jugement, procès, responsabilité et punition, dans un registre qui s'inscrit encore une fois dans le champ lexical de la répression. Ainsi, le 31 mars, après seulement 15 jours de quarantaine, le pays compte déjà 36000 citoyens en garde à vue ${ }^{14}$ contre 1065 cas déclarés de COVID-19 ${ }^{15}$. Cette disproportion entre le nombre de contaminations et le nombre de citoyens arrêtés pour n'avoir pas respecté les consignes laisse transparaître le poids des choix réalisés, qui se traduisent dans l'espace urbain par un contrôle constant d'ordre militaire.

Les derniers jours du mois de mars, un débat se déclenche autour de la loi n 31012, dite "de protection policière " (approuvée le 28 mars en plein état d'urgence). Cette loi assure l'immunité aux policiers comme aux militaires qui, pendant l'exercice de leurs fonctions, blesseront ou tueront quelqu'un. L'approbation de cette loi, surtout dans un 
moment de crise comme celui-ci, a immédiatement soulevé des préoccupations dans la communauté internationale. La commission des droits humains du bureau latinoaméricain de l'ONU a demandé à l'État péruvien de revoir la loi ${ }^{16}$. Parallèlement, sa promulgation témoigne de l'importante confiance accordée à l'armée pour la gestion de cette crise au Pérou. Selon le rapport de l'INEI (Institut national de Statistique et d'Informatique $)^{17}$, début 2020, l'armée apparait en quatrième position parmi les institutions auxquelles les Péruviens font les plus de confiance. Ainsi, face à la COVID-19, les forces armées apparaissent être efficaces et structurées, avec une chaîne de commandement claire, en contraste avec une bureaucratie étatique contrôlée par une classe politique impliquée dans de graves actes de corruption.

Si on perçoit une présence massive de l'armée dans l'espace public, celle-ci joue aussi un rôle central à différentes échelles de la gestion de crise au sein de l'organisation gouvernementale. Sur ce point, la composition du «Comando de Emergencia COVID19 $»^{18}$ semble révélatrice. Il s'agit d'un organe spécial, créé le $1^{\mathrm{er}}$ avril 2020 pour développer une stratégie performante de gestion de crise. Au moment de sa création, cette équipe se compose exclusivement d'acteurs de l'administration sanitaire et de représentants de l'armée ${ }^{19}$.

En même temps, l'armée est l'acteur principal qui a été mobilisé par le gouvernement à toutes les échelles, ainsi que pour le réaménagement du territoire face à la crise. Grâce à son intervention, de nouvelles infrastructures de soins ont été construites très rapidement afin de pouvoir prendre en charge le plus grand nombre de malades de COVID-19. Les interventions ont été concentrées dans la capitale, qui compte aujourd'hui presque un tiers de la population nationale (selon les données de l'INEI en 2018, environ 9 millions d'habitants, 10 millions en comptant la région de Callao). La direction générale de Gestion de risque de désastre et de défense nationale en santé (DIGERD) a donné priorité à l'installation des hôpitaux de campagne (c'est-à-dire des modules isolés pour recevoir des cas de COVID-19) dans les cinq hôpitaux majeurs de la métropole de Lima (Cayetano Heredia, Sergio Bernales, Hipólito Unanue, Villa El Salvador et Dos de Mayo) et dans l'aéroport international Jorge Chávez ${ }^{20}$. De la même manière, elle a transformé en hôpital la Villa Panamericana ${ }^{21}$ pour traiter jusqu'à 3000 cas de la nouvelle maladie.

Malgré la réforme adoptée en 2011, qui a réaffirmé la volonté de décentraliser aux niveaux régionaux et municipaux la gestion des risques, à travers des soutiens techniques et financiers, le Pérou semble faire reposer principalement sur les épaules de l'armée l'organisation de la phase de réponse à la crise, d'une manière très centralisée, comme dans le cas de El Niño en 2017 ou lors du séisme de Pisco de l'année 2007 (Delaître et al., 2018). Au moment du Niño, face à la défaillance des gouvernements locaux dans l'organisation des secours, le gouvernement central avait décidé d'attribuer le commandement du Centre national des opérations d'urgence au ministère de la Défense, modifiant considérablement l'organigramme du Système national de gestion du risque de désastres établi en 2011. Cette adaptation des procédures rappelle les organisations antérieures aux années 2000, à matrice militaire (Delaître et al., 2018).

"L'histoire montre qu'au Pérou, le paradigme de l'urgence dans la gestion des catastrophes et l'acteur militaire sont particulièrement liés» (La Red, 1996). Ainsi, lorsque en 1961 nait le premier organe explicitement dédié à la « défense contre les accidents publics » (les tremblements de terre, les inondations, les sécheresses, etc.), 
bien que multisectoriel et multi-institutionnel, il est présidé par un général de l'armée nommé sur proposition du ministère de la Défense.

\section{Conclusion}

Le Président péruvien, qui se trouve dans la difficulté de n'être pas soutenu par la classe politique au pouvoir, notamment au sein du Congrès, a trouvé dans la pandémie l'opportunité de démontrer et d'affirmer davantage son statut de porte-voix de l'unité nationale face à l'adversité ; il assume le rôle de protecteur du peuple péruvien. En consolidant son lien avec la population à travers un dialogue constant, il semble renforcer son soutien populaire. Parallèlement, les relations de pouvoir au sein du Pérou se compliquent lorsque, début août, le Congrès refuse le nouveau cabinet de ministres proposé par Vizcarra. Il devra en proposer un autre pour avoir l'approbation. À la suite de cet événement, le Président fera appel à l'unité politique : «Ce n'est pas le moment de la division, mais de l'unité et du consensus. Le devoir des Péruviens et des autorités est bien au-dessus de toutes différences circonstancielles. Notre obligation envers la patrie signifie également tourner la page des différences (politiques) et continuer à travailler pour tous les Péruviens $»^{22}$. La crise parait être instrumentalisée par le gouvernement à la recherche d'un consensus politique. En même temps, le nouveau cabinet des ministres élu est dirigé par un ancien militaire, qui était auparavant ministre de la Défense, en qualité de premier ministre (Walter Martos). La dimension militaire rester ainsi présente sous différents aspects de la gestion de crise et à différents niveaux de gouvernance.

Certains analystes critiquent les États ibéro-américains qui, comme le Pérou, utilisent leurs forces de sécurité dans des tâches non militaires. Ils estiment que ces tâches devraient être prises en charge par des moyens civils des États, alors même que ceux-ci n'ont souvent pas assez de ressources pour le faire. Ces mêmes chercheurs sont de même assez critiques concernant l'implication de l'armée dans d'autres types de contextes hors conflit, en soulignant que ceci pourrait fausser le concept de Défense. Ainsi, ils questionnent l'identité de l'armée, dont la mission principale devrait être la défense de la souveraineté nationale (Talaván, 2003 ; Kruijt, 2012 ; Koonings et Kruijt, 2014 ; De la Torre Rotta, 2016).

Une crise permet de mettre en lumière les conditions ordinaires du fonctionnement des sociétés, justement quand celui-ci bascule dans une dimension extraordinaire. Ainsi, un paradigme de gestion de crise, une fois mis en œuvre, est-il indissociable des caractéristiques historiques et socio-culturelles propres à la société dans laquelle il se développe. Les enseignements de la matrice militaire de ce modèle de gestion de crise quant à la place de l'armée dans la société péruvienne restent à tirer.

\section{BIBLIOGRAPHIE}

Calderón J., 2016 [2nda ed.]. La ciudad ilegal. Lima en el siglo XX. Lima, Editorial Punto Cardinal. 
Carroué L., 2020. Mondialisation et démondialisation au prisme de la pandémie de Covid-19. Le grand retour de l'espace, des territoires et du fait politique. Géoconfluences [En ligne], mai 2020. URL: http://geoconfluences.ens-lyon.fr/actualites/eclairage/covid19-mondialisationdemondialisation

Chartier A., Rivière M., 2018. Les effets secondaires de l'aide au développement : Comment l'aide stimule les jeux de pouvoir à Madagascar. Revue internationale des études du développement [En ligne], vol. 234, n 2, p. 123-150. URL: https://doi-org.ezpaarse.univ-paris1.fr/10.3917/ried. 234.0123

Darbon D. (dir.), 2009. La Politique des modèles en Afrique. Simulation, dépolitisation et appropriation. Paris - Bordeaux, Éditions Karthala.

De la Torre Rotta, A.G., 2016. ¿Nuevos roles de las Fuerzas Armadas en el Perú? In coll., El nuevo rol de las Fuerzas Armadas en Bolivia, Brasil, Chile, Colombia, Ecuador y Perú. Pontificia Universidad Católica del Perú, Instituto de Estudios Internacionales (IDEI), Lima - Konrad Adenauer Stiftung, p. 171-197.

Delaître M., Sierra A., Barroca B., Vargas Florez J., 2018. De la lecture d'une crise à celle de la résilience territoriale : le phénomène el Niño 2017 au Pérou vu depuis la capitale [En ligne]. Published by ISTE Ltd. London, UK - openscience.fr. URL: https://www.openscience.fr/IMG/pdf/ iste_uris18v2n1_3.pdf

Fabiani J., Theys J., 1987. La société vulnérable. Evaluer et maitriser les risques. Paris, Presses de l'école Normale Supérieure.

Gilbert C., 1992. Le pouvoir en situation extrême - Catastrophes et Politique. Editions L'Harmattan, $272 \mathrm{p}$.

Koonings K., Kruijt D., 2002. Fuerzas Armadas y política en América Latina: perspectivas futuras. Iberoamericana. América Latina, España, Portugal [En ligne], n 8, p.7-22:. URL: https:// journals.iai.spk-berlin.de/index.php/iberoamericana/article/view/570; DOI: https://doi.org/ 10.18441/ibam.2.2002.8.7-22

Kruijt D., 2012. Las fuerzas armadas en América Latina, antes y hoy. Ciencia Politica, $\mathrm{n}^{\circ} 14$, p. $94-112$.

La Red de Estudios Sociales en Prevención de Desastres en América Latina, 1996. Estado, sociedad y gestión de los desastres en américa latina. En busca del paradigma perdido. Allan Lavell, Eduardo Franco (Editores)

Lagadec P., 1991. La gestion des crises : Outils de réflexion à l'usage des décideurs. McGraw-Hill.

Lussault M. (réalisateur), 2020. Chronique Géovirale [En ligne sur You Tube]. École urbaine de Lyon, série de chroniques vidéos mises en ligne entre le 21 mars et le 11 mai 2020. URL: https:// medium.com/anthropocene2050/chroniques-g\%C3\%A9o-virales-e144c57db628

Mesclier É., 2020. Pérou : les ombres de l'émergence économique sous les projecteurs de la Covid-19. COVIDAM : la Covid-19 dans les Amériques [En ligne], Institut des Amériques, 24 juin 2020. URL: https://covidam.institutdesameriques.fr/perou-les-ombres-de-lemergence-economiquesous-les-projecteurs-de-la-covid-19/

Mizrahi D., 2019. Martín Vizcarra, el líder menos pensado: del "exilio" en Canadá a la lucha contra la "casta política" en Perú. Infobae, América Latina [En ligne], 6 octobre 2019. URL: https:// www.infobae.com/america/america-latina/2019/10/06/martin-vizcarra-el-lider-menospensado-del-exilio-en-canada-a-la-lucha-contra-la-casta-politica-en-peru/ 
Peru21. Site de presse locale (consulté le 4 avril 2020). URL: https://peru21.pe/peru/coronavirusen-peru-hombres-y-mujeres-podran-salir-en-dias-intercalados-los-domingos-hay-restriccionpara-todos-hoy-02-de-abril-en-directo-vivo-online-ultimas-noticias-muertos-infectadoscontagiados-ultimo-minuto-martin-vizcarra-minsa-minuto-a-minuto-del-covid-19-alerta-sosestado-de-emergencia-cuarentena-online-noticia/?fbclid=IwAR2B-q3-Z7T00crro_1QOYKHgvVdsRs54AZ8vHZ45E5mbBgYp2gb5N9rig

Peyroux É., 2012. Circulation internationale et construction sociale d'un « modèle » de gestion des services urbains : les city improvement districts à Johannesburg. L'Espace géographique [En ligne], vol. 41, n 1, p. 68-81. URL: https://doi-org.ezpaarse.univ-paris1.fr/10.3917/eg.411.0068 - DOI : https://doi-org.ezpaarse.univ-paris1.fr/10.3917/eg.411.0068

Revêt, S., 2018. Les coulisses du monde des catastrophes «naturelles ». Paris, Éditions de la Maison des sciences de l'homme [Edition en ligne sur OpenEdition - URL: https://books.openedition.org/ editionsmsh/11688?lang=fr]

Sierra A., 2004. Risques climatiques et enjeux politiques : le cas de la crise équatorienne liée au phénomène du Nino en 1997. In Gilber D. (dir.), Espaces tropicaux et risques. Du local au global, Journées de géographie tropicale Orléans, 24, 25 et 26 septembre 2003. IRD - Presses Universitaires d'Orléans, p. 84-94.

Sierra A., 2007. Les enjeux géopolitiques de l'institutionnalisation de la gestion du risque « naturel » en Amérique latine. Le cas du Costa Rica, de la Colombie et de l'Équateur. Outre-Terre, $\mathrm{n}^{\circ} 18, \mathrm{p} .37-54$.

Sierra A., 2015. La capitale-risque ou comment le statut de capitale participe à construire le risque. Géocarrefour, vol. 90, n² 2, p. 173-182.

Sierra A., 2020. Déclencher la crise pour anticiper la catastrophe ? Trois pays andins face à la pandémie, COVIDAM : la Covid-19 dans les Amériques [En ligne], Institut des Amériques, 26 avril 2020. URL: https://covidam.institutdesameriques.fr/declencher-la-crise-pour-anticiper-lacatastrophe-trois-pays-andins-face-a-la-pandemie/

Talaván M. L., 2003. Las relaciones cívico-militares en el Perú actual. Cuadernos de estrategia, no 123 , p. 189-218.

Toche Medrano E., 2008. Guerra y democracia: los militares peruanos y la construcción nacional [En ligne]. Lima, Desco - CLACSO. URL: http://bibliotecavirtual.clacso.org.ar/clacso/becas/ 20120419125101/medrano.pdf

\section{NOTES}

1. Selon le site officiel du ministère de la Santé péruvien : 317993 cas dans la métropole de Lima, et 30432 dans la région du Callao. Données du 15/09/2020. URL: https://covid19.minsa.gob.pe/ sala_situacional.asp

2. La dengue au Pérou affecte principalement les zones amazoniennes.

3. Traduction de l'auteure, voici la version dans la langue d'origine : «acompañado del pleno del consejo de ministros, de representantes de la asamblea general de gobiernos regionales y del presidente de la asociación de municipalidades del Perú ». Discours à la Nation du 15/03/2020. URL: https:// www.youtube.com/watch?v=FoNmdCGZ4f0\&ab_channel=PresidenciaPer\%C3\%BA

4. Traduction de l'auteure, voici la version dans la langue d'origine: «Países que han asumido responsablemente su tarea entorno a esta enfermedad han salido airosos. Los países que no lo han hecho, están lamentando cantidades importantes de pérdidas humanas, cantidades muy significativas de perdida 
económica, pero lo más importante, con futuro incierto ». Discours à la Nation du 16/03/2020. URL: https://www.youtube.com/watch?v=_K75UYGj3go\&ab_channel=CarlaCastro

5. Traduction de l'auteure, voici la version dans la langue d'origine : "La policía nacional y la fuerzas armadas adoptarán las medidas para garantizar los servicios públicos. Durante el estado de emergencia, quedan restringidos los derechos constitucionales relativos a la libertad y la seguridad personales, y la inviolabilidad del domicilio y la libertad de reunión y de tránsito en el territorio nacional». Discours à la Nation du 15/03/2020. URL: https://www.youtube.com/watch? $\mathrm{v}=$ FoNmdCGZ4fo\&ab_channel=PresidenciaPer\%C3\%BA

6. Transparency International le cite parmi les dix anciens chefs d'États les plus corrompus des vingt dernières années.

7. Il s'agit d'une multinationale brésilienne très présente dans toute l'Amérique latine, qui a été impliquée dans de multiples scandales de corruption au Brésil et ailleurs sur le continent.

8. Traduction de l'auteure, voici la version dans la langue d'origine : «El estado está cumpliendo, con las instituciones públicas, está cumpliendo con las familias y personas vulnerables. Muchos empresarios responsables están cumpliendo también. Lo que pedimos es que todos cumplamos, depende de nosotros. Podemos enfrentar de manera satisfactoria esta enfermedad tan difícil, si cumplimos todos responsablemente. Yo estoy seguro, confío plenamente en la responsabilidad de todos los peruanos que vamos a salir de este problema de manera satisfactoria ». Discours à la Nation 16/03/2020.URL: https://www.youtube.com/watch?v=_K75UYGj3go\&ab_channel=CarlaCastro

9. Site consulté le $1^{\text {er }}$ avril 2020. URL: https://peru21.pe/peru/coronavirus-en-peru-toque-dequeda-ira-de-4-pm-a-5-am-en-tumbes-piura-lambayeque-la-libertad-y-loreto-hoy-30-de-marzoen-directo-vivo-online-ultimas-noticias-muertos-infectados-contagiados-ultimo-minuto-martinvizcarra-minsa-minuto-a-minuto-del-covid-19-noticia/

10. Site consulté le 2 avril 2020. URL: https://rpp.pe/peru/actualidad/estado-de-emergenciacoronavirus-martin-vizcarra-anuncio-diferentes-dias-de-circulacion-para-hombres-y-mujeresnoticia-1255993

11. Par le Decreto Supremo $N^{\circ} 004-2020$ DE.

12. Site consulté le 4 avril 2020. URL: https://rpp.pe/politica/gobierno/coronavirus-covid-19martin-vizcarra-no-es-que-al-dia-siguiente-del-estado-de-emergencia-se-retira-a-las-fuerzas-

armadas-noticia-1255444?ref=rpp

13. Site été consulté le 4 avril 2020. Traduction de l'auteure, extrait original en espagnol. URL: https://actualidad.rt.com/actualidad/347999-vizcarra-peru-multas-carcel-incumplircuarentena-coronavirus

14. Site consulté le 4 avril 2020. URL: https://larepublica.pe/sociedad/2020/03/31/coronavirusen-peru-mas-de-36-000-detenidos-desde-que-inicio-el-estado-de-emergencia-video/ 15. Site consulté le 4 avril 2020. URL: https://larepublica.pe/sociedad/2020/03/31/coronavirusperu-en-vivo-minuto-a-minuto-ultimas-noticias-hoy-martes-31-de-marzo-2020-casosconfirmados-covid-19-muertes-infectados-y-contagiados-en-estado-de-emergencia-atmp/ 16. Sites consultés le 4 avril 2020. URL: https://rpp.pe/peru/actualidad/onu-pide-revisar-ley-deproteccion-policial-por-crear-espacios-de-impunidad-noticia-1255849 - URL : https:// elcomercio.pe/politica/ley-de-proteccion-policial-la-controversia-por-la-norma-y-laspropuestas-tras-su-publicacion-estado-de-emergencia-coronavirus-noticia/ - URL: https:// larepublica.pe/politica/2020/04/01/epaf-ley-de-proteccion-policial-es-un-error-en-un-paisposconflicto-con-muertos-y-desparecidos/

17. INEI, 2020. Perú: percepción ciudadana sobre gobernabilidad, democracia y confianza en las instituciones,semestre: octubre 2019 - marzo 2020. Informe técnico, nº2, Mayo 2020 [En ligne]. URL:

http://m.inei.gob.pe/media/MenuRecursivo/boletines/ informe_de_gobernabilidad_may2020.pdf

18. Site consulté le 4 avril 2020. URL: https://larepublica.pe/sociedad/2020/04/01/coronavirusen-peru-martin-vizcarra-anuncia-la-creacion-del-comando-de-emergencia-covid-19/ 
19. Pilar Mazzeti (Ministère de Salut-MINSA), Oscar Ugarte (EsSalud) Victor Bocángel (MINSA), Mario Cacho (Marine de guerre), Edwin Córdova (Armée Aérienne) et Fernando Tapia (Armée Péruvienne), Jorge Luis Quiroz (Police Nationale -PNP) y Alejandro Lampe (pour représenter les cliniques privées).

20. Site consulté le 4 avril 2020. URL: https://www.minsa.gob.pe/digerd/index.asp? op $=61 \&$ not $=480$

21. Résidence pour les athlètes des Jeux Panaméricains en 2019. Site consulté le 31 mars 2020. URL: https://rpp.pe/multideportes/panamericanos/coronavirus-la-villa-panamericana-un-granlegado-avanzan-los-trabajos-para-el-hospital-mas-grande-del-pais-noticia-1253293

22. Traduction de l'auteure, voici la version dans la langue d'origine : « No es un momento para la división, si no para la unidad y el consenso. El deber que tenemos los peruanos y las autoridades está muy por encima de cualquier diferencia circunstancial. Nuestra obligación ante la patria significa también voltear la página de las diferencias y continuar trabajando en pro de todos los peruanos. » Site visité le 10 septembre 2020. URL: https://www.france24.com/es/20200806-peru-vizcarra-gabineteministros-pandemia

\section{RÉSUMÉS}

Cet article propose une lecture de la gestion de crise engendrée par l'épidémie de COVID-19 au Pérou, observée depuis Lima. Il offre une réflexion sur la manière dont le paradigme de gestion a été façonné par le contexte péruvien, au fur et à mesure de la diffusion de l'information. Ainsi, nous analysons la construction de cette stratégie de réponse, en mettant l'accent sur le discours qui la définit. Par cette étude, nous cherchons à faire émerger les enjeux de pouvoir que cette situation de crise révèle, en nous focalisant sur l'implication de l'armée.

This article offers an interpretation of the crisis management caused by the COVID-19 epidemic in Peru, observed from Lima. This analysis offers a reflection about the transformation of the management paradigm of the crisis by the Peruvian context, according to the process of information production and diffusion. Thus, we examine the construction of this response strategy by an analysis of the official speech that defines it. Through this study, we seek to bring out the issues of power that this crisis situation reveals, by focusing on the involvement of the army.

\section{INDEX}

Mots-clés : Pérou, crise, COVID-19, réponse, militarisation

Keywords : Perú, crisis, COVID-19, response, militarization

\section{AUTEUR}

\section{IRÈNE VALITUTTO}

Irène Valitutto, irene.valitutto@gmail.com, est doctorante de l'université de Paris 1 Panthéon Sorbonne et associée à l'UMR Prodig. 